\title{
A Cyclic GMP Analog Decreases the Currents Underlying Bursting Activity in the Aplysia Neuron R15
}

\author{
Edwin S. Levitan a and Irwin B. Levitan \\ Graduate Department of Biochemistry, Brandeis University, Waltham, Massachusetts 02254
}

Bath application of 8-parachlorophenylthio-cyclic GMP (8pcpt-cGMP) has been shown to increase the number of action potentials per burst in the Aplysia neuron R15. Here we report that 8-pcpt-cGMP can eventually inhibit R15's bursting activity and cause the cell to exhibit slow tonic spiking activity. This action is preceded by decreases in spike frequency and in the amplitude of the interburst hyperpolarization. The ionic mechanism underlying these various changes in burst pattern was investigated using voltage clamp. 8-pcptcGMP reduces steady-state inward current, and this effect is blocked by manganese but is unaffected by internal EGTA or elevation of extracellular potassium. Dopamine, which decreases the subthreshold calcium current in R15, diminishes the change in current induced by 8-pcpt-cGMP. 8-pcpt-cGMP also reduces the spike-evoked calcium-dependent inward and outward currents known to underlie burst generation. These data lead us to conclude that 8-pcpt-cGMP acts directly to reduce the steady-state subthreshold calcium current $\left(I_{\mathrm{ca}}\right)$. This decrease in $I_{\mathrm{ca}}$ may also indirectly reduce the calcium-dependent currents. It is suggested that 8-pcpt-cGMP produces its effects on bursting by decreasing both the inward and outward currents required for generating bursting behavior, and thus effectively dampens bursting until the cell is in a beating state. It is also shown that 8-pcptcGMP dramatically alters $\mathrm{R} 15$ 's responses to depolarizing and hyperpolarizing stimuli. Thus, this cyclic GMP analog alters not only the cell's intrinsic activity, but also its response to external inputs.

Bursting electrical activity is produced by many neurons and endocrine tissues (Rapp, 1979; Carpenter, 1982). The importance of bursting in the nervous system is at least 2-fold. First, the rhythmic outputs of bursting cells serve as timers or pacemakers in central pattern generators (Selverston and Moulins, 1985). Bursting activity is also found in many neurosecretory cells presumably becausc it represents the optimal activity for producing secretion and synaptic release (Gillary and Kennedy, 1969; Dutton and Dyball, 1979). Induction and modulation of bursting activity by synapses and transmitters have been found in both vertebrate and invertebrate nervous systems (Parnas et

\footnotetext{
Received Jan. 9, 1987; revised Sept. 28, 1987; accepted Sept. 29, 1987.

We are grateful to Dr. W. B. Adams for providing us with his computer model and to Dr. R. Kramer for his advice. We also thank Drs. R. Kramer and E. Marder for their critical comments on an earlier version of the manuscript. This work was supported by NINCDS Grant NS17910 (to I.B.L.).

Correspondence should be addressed to Irwin B. Levitan at the above address.

a Present address: MRC Molecular Neurobiology Unit, University of Cambridge Medical School, Hills Road, Cambridge CB2 2QH, England.

Copyright (C) 1988 Society for Neuroscience $0270-6474 / 88 / 041162-10 \$ 02.00 / 0$
}

al., 1974; Wilson and Wachtel, 1978; Grace and Bunney, 1984; Marder and Eisen, 1984; Roth et al., 1984; Dekin et al., 1985; Harris-Warrick and Flamm, 1986; Ruben et al., 1986). Despite the importance and prevalence of bursting neurons, however, in most cases little is known about the ionic mechanisms underlying their modulation. This is because few of these cells are well suited for voltage-clamp study.

The neurosecretory Aplysia cell R15 intrinsically generates robust bursting activity (Alving, 1968). Voltage-clamp studies have elucidated the ionic mechanism for producing bursting activity in this and other Aplysia neurons. It has been demonstrated that bursting activity is produced by the action of calcium current $\left(I_{C_{a}}\right)$ and calcium-dependent currents (Gorman et al., 1982; Adams, 1985; Adams and Levitan, 1985; Kramer and Zucker, 1985a, b). Likewise, the actions of modulatory transmitters and cyclic nucleotides on R 15 have been studied in detail (see Adams and Benson, 1985). For example, bath application of a membrane-permeable cyclic GMP analog (8-pcpt-cGMP) increases the number of action potentials per burst produced by cell R15 (Levitan and Norman, 1980).

Here, we report that 8-pcpt-cGMP can disrupt R15's bursting activity and cause the cell to produce tonic spiking or beating activity. Voltage-clamp studies reveal that 8-pcpt-cGMP reduces the steady-state subthreshold $I_{\mathrm{Ca}}$. Voltage-clamp analysis further demonstrates that both the inward and outward calciumdependent currents underlying bursting activity are also decreased by 8 -pcpt-cGMP. It is suggested that the reduction of $I_{\mathrm{Ca}}$ and of calcium-dependent currents leads to a dampening of bursting that culminates in beating activity.

\section{Materials and Methods}

Materials and methods were similar to those reported in Levitan and Levitan (1988). The 8-pcpt-cGMP was synthesized as described by Miller et al. (1973), and was bath applied at a concentration of $1.7 \mathrm{~mm}$ for $10 \mathrm{~min}$ unless otherwise stated. Its effects were reversible upon washing. All experiments reported here were replicated at least 3 times, and the data shown are representative.

Simulation of bursting activity under voltage clamp consisted of presenting trains of $10-\mathrm{msec}-$ long $100 \mathrm{mV}$ depolarizing pulses, separated by $0.5-2 \mathrm{sec}$, alternated with long periods of inactivity at the holding potential of $-50 \mathrm{mV}$. Typically, a burst cycle of $40 \mathrm{sec}$ was used. Currents were typically filtered at $30 \mathrm{~Hz}$.

\section{Results}

\section{8-pcpt-cGMP dampens bursting activity produced by $R 15$}

Shown in Figure $1 A$ is the basal bursting activity of cell R15. Upon bath application of 8-pcpt-cGMP, the first change in activity seen is an increase in the interval between action potentials within the burst (Fig. 1B). Later, the interburst hyperpolarizations become shallower (Fig. 1, $C, D$ ). Long bursts with many action potentials are produced, as reported previously (Levitan 
A

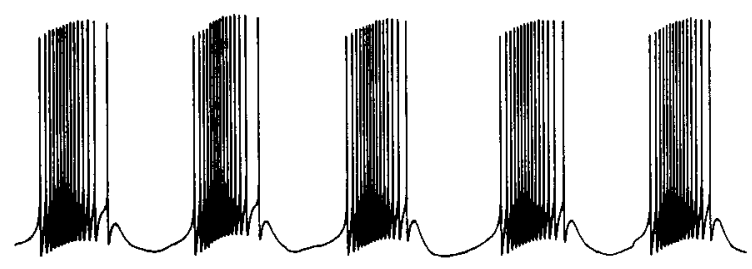

B

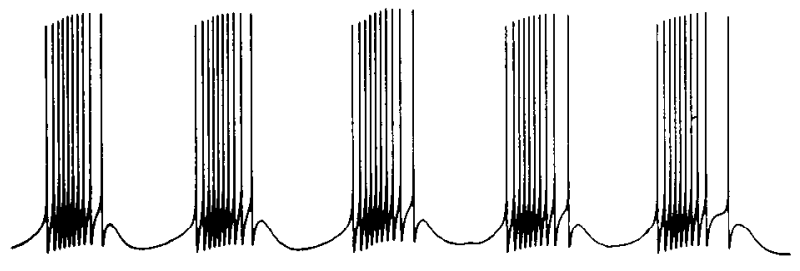

C

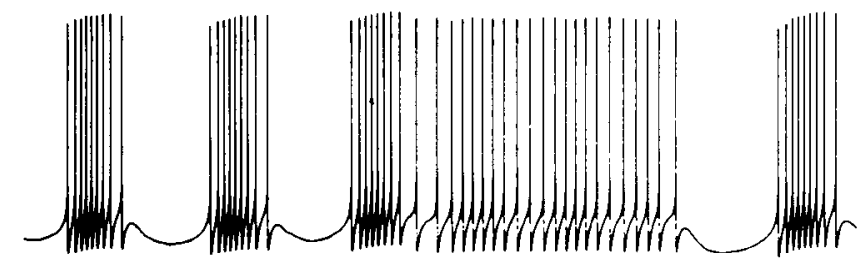

D

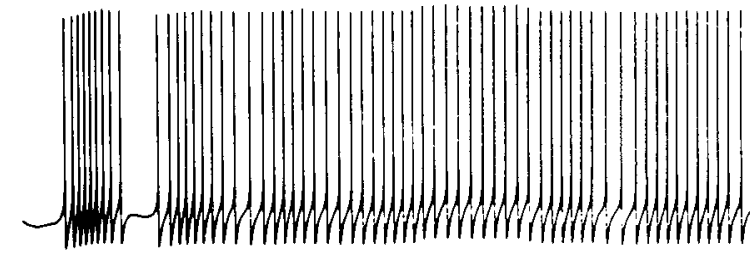

E

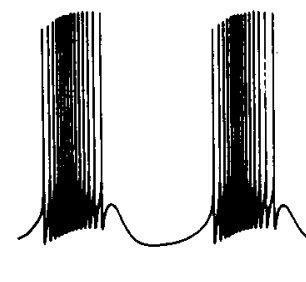

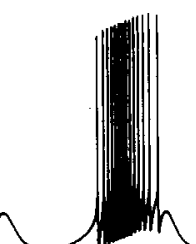

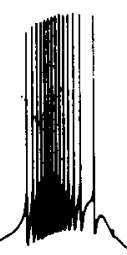

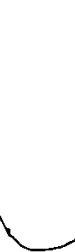

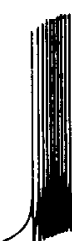

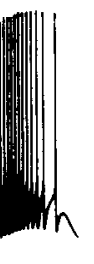

Wash $20 \mathrm{mV}$
$13 \mathrm{Min}$

8-pCpt-cGMP
$18 \mathrm{Min}$

8-pcpt-cGMP and Norman, 1980), but spiking occurs at a very low frequency (Fig. 1, C, D). Eventually, the cell stops bursting and instead produces slow tonic spiking or beating activity (Fig. $1 D$ ). The effects of 8-pcpt-cGMP are reversible upon washing (Fig. 1E). Thus, 8-pcpt-cGMP, like cyclic AMP under some conditions (Levitan and Levitan, 1988), can convert R15 from a burster to a beater. Unlike the effect of cyclic AMP, however, this is accompanied by a decrease in action potential frequency. Thus, it does not appear that 8-pcpt-cGMP acts simply by depolarizing R15.

The effects of 8-pcpt-cGMP take many minutes to develop and nearly an hour to reverse upon washing. They are not mimicked by bath application of equivalent concentrations of cyclic GMP or 8-bromo-cyclic GMP, both of which are less membrane-permeable than 8-pcpt-cGMP (data not shown). Furthermore, dibutyryl-cGMP, a compound known for not activating cyclic GMP-dependent protein kinase (Meyer and Miller,
Figure 1. Effect of $0.85 \mathrm{~mm} 8$-pcptcGMP on the bursting activity of R15. $A$, Control activity. $B$, After 13 min perfusion with 8-pcpt-cGMP, a decrease in spike frequency during the burst is seen. $C$, After $18 \mathrm{~min}$, a depolarization of the interburst hyperpolarization is evident and long bursts become apparent. $D$, Continuous record from $C$. The final burst lasted many minutes. $E$, On washout the effects of 8-pcpt-cGMP reversed.
1974) or cyclic GMP-gated ion channels in the vertebrate photoreceptor (Zimmerman et al., 1985), does not produce effects similar to 8-pcpt-cGMP, despite the fact that it is membrane permeable (data not shown). These data suggest that 8 -pcptGMP acts intracellularly at a specific site to modulate bursting activity.

\section{8-pcpt-cGMP decreases the voltage-gated calcium current}

Voltagc-clamp studies first addressed whether 8-pcpt-cGMP affects subthreshold currents that are known to play a part in burst production (Adams, 1985; Adams and Levitan, 1985; Kramer and Zucker, 1985a, b). Shown in Figure $2 A$ is a current versus voltage $(I-V)$ curve from a cell before and after exposure to 8-pcpt-cGMP. 8-pcpt-cGMP induces an outward voltage-gated current, which usually begins to activate between -75 and -60 $\mathrm{mV}$. No reversal is seen, even at voltages very much more negative than the $\mathrm{K}^{+}$equilibrium potential (i.e., $-75 \mathrm{mV}$ ). The 
Figure 2. 8-pcpt-cGMP reduces the subthreshold inward current. $A$, Current versus voltage $(I-V)$ curve from a cell before $(\square)$ and after $(\triangle)$ a $10 \mathrm{~min}$ perfusion with $1.7 \mathrm{~mm} 8$-pcpt-cGMP. Currents were evoked by $0.5 \mathrm{sec}$ pulses from $-70 \mathrm{mV}$. B, Current (lower trace) evoked by depolarization (upper trace) from $-52 \mathrm{mV}$ in low-calcium SW. $C$ Same cell as $B$ after a $10 \mathrm{~min}$ exposure to $1.7 \mathrm{~mm} 8$-pcpt-cGMP.
A

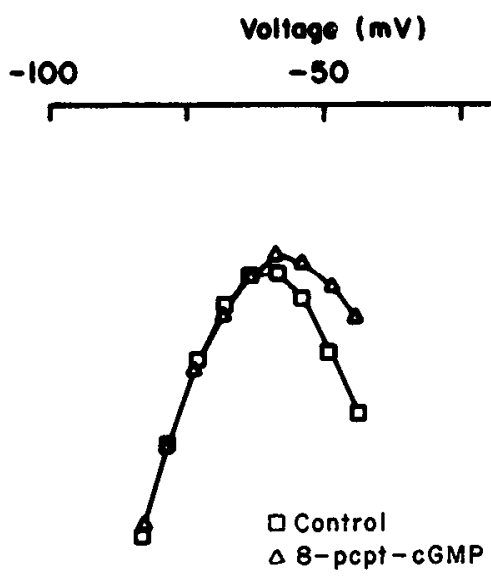

B

0

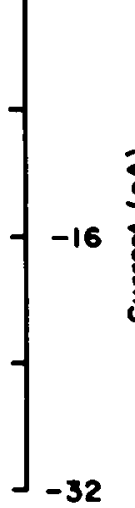

C

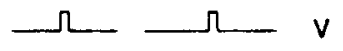

Control 8-pcpt-cGMP

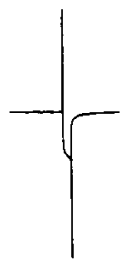

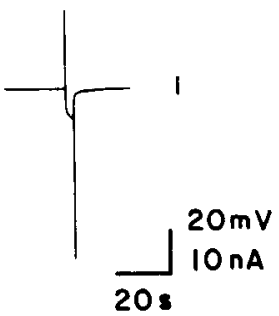

8-pcpt-cGMP-induced current could be due to an increase in outward potassium or chloride currents, or to a decrease in the resting inward current, which is known to be carried by calcium (Gorman et al., 1982; Lewis et al., 1984).

This net outward current is also seen under conditions that dramatically reduce synaptic activity but leave the subthreshold $I_{\mathrm{Ca}}$ partially intact. Shown in Figure $2 B$ is the current evoked by a small depolarizing voltage pulse in a cell bathed in a low Ca SW [see Levitan and Levitan (1988) for the ionic composition of this and other bathing solutions]. Applying 8-pcptcGMP shifts the holding current at $-52 \mathrm{mV} 5 \mathrm{nA}$ outward and decreases the voltage-gated current by $43 \%$ (Fig. $2 C$ ). Thus, 8-pcpt-cGMP induces net outward current by acting directly on R15.

Analysis of the tail currents that follow depolarizing pulses favors the hypothesis that the cyclic GMP analog decreases resting $I_{\mathrm{Ca}}$. If 8-pcpt-cGMP increased an outward potassium or chloride current, one would expect that the outward tail current following a depolarizing pulse would become larger in the presence of 8-pcpt-cGMP. In contrast, we have found that 8-pcptcGMP decreases the slow outward tail current evoked by long subthreshold depolarizing pulses (see arrows in Fig. 3, $A, B$ ). This outward tail current is due to inactivation of resting inward $I_{\mathrm{Ca}}$ by accumulation of internal calcium during the depolarizing pulse (Adams and Levitan, 1985; Kramer and Zucker, 1985b).

The extent of the decrease in the outward tail current is similar to the decrease in the voltage-gated inward current during the pulse. For example, in the representative cell shown in Figure $3, A$ and $B$, the voltage-gated inward current during the pulse is reduced by $42 \%$, while the slow outward tail current following the pulse is reduced by 50\%. 8-pcpt-cGMP also reduces the slow outward tail current that follows trains of short suprathreshold pulses (see below). These results support the hypothesis that 8-pcpt-cGMP decreases $I_{\mathrm{Ca}}$ and, therefore, decreases the calcium-dependent inactivation of resting $I_{\mathrm{Ca}}$ underlying the slow outward tail current.

To further test whether 8-pcpt-cGMP acts on $I_{\mathrm{Ca}}$, calcium was substituted with manganese. Shown in Figure $3 A$ is the basal voltage-gated subthreshold inward current in normal saline. Upon application of 8-pcpt-cGMP this current decreases (Fig. $3 B$ ). After washing out the 8-pcpt-cGMP, calcium was substituled with manganese. This blocks the subthreshold calcium current (Fig. 3C). Application of 8-pcpt-cGMP in the presence of manganese produces no outward current (Fig. 3D). Thus, blocking $I_{\mathrm{Ca}}$ with manganese also blocks the 8-pcpt-cGMP-induced voltage-gated outward current. This result further suggests that 8-pcpt-cGMP acts on $I_{\mathrm{Ca}}$ or on a calcium-dependent current.

To differentiate between these 2 possibilities, calcium-activated currents were blocked by loading $\mathrm{R} 15$ with the calcium chelator EGTA. Depolarization still evokes the voltage-gated $I_{\mathrm{Ca}}$, although the calcium-dependent outward tail current is blocked (Fig. 3E). Application of 8-pcpt-cGMP continues to reduce the voltage-gated inward current (Fig. $3 F$ ). Thus, 8-pcptcGMP cannot be acting by increasing a calcium-activatcd outward current such as a calcium-activated potassium current. This also demonstrates that 8-pcpt-cGMP does not act by increasing calcium-dependent inactivation of the calcium current.

The data presented thus far do not eliminate the possibility that 8-pcpt-cGMP activates a manganese blockable voltagegated potassium current. In fact, a potassium current that is not activated by intracellular calcium has been shown to be sensitive to divalent cations (Paupardin-Tritsch et al., 1981). To rule out activation of potassium channels by 8-pcpt-cGMP, the effects of 8-pcpt-cGMP were compared in normal $(10 \mathrm{mM})$ and elevated $(25 \mathrm{~mm})$ potassium. Reducing the driving force for potassium approximately 2 -fold does not alter the 8-pcpt-cGMPinduced outward current (Fig. 4). Thus, 8-pcpt-cGMP does not appear to be acting by increasing potassium current.

Dopamine has been shown to decrease axonal $I_{\mathrm{Ca}}$ in R 15 (Lewis et al., 1984). If dopamine and 8-pcpt-cGMP act on the same ion channels, then dopamine should occlude the 8-pcptcGMP effect. Figure 5 shows the amplitude of the outward current induced by 8-pcpt-cGMP at different membrane potentials. These data were obtained by subtracting the $I-V$ curve in the presence of 8-pcpt-cGMP from the $I-V$ curve after washing away the 8-pcpt-cGMP. The cell was then again exposed to 8-pcpt-cGMP but this time in the presence of dopamine, and again a difference curve is shown. This difference current is due in part to the desensitization of the effects of saturating dopamine during the long time course of the experiment. The recovery of dopamine-blocked $I_{\mathrm{Ca}}$ (due to desensitization) produces an outward difference current that sums with the 8-pcptcGMP-induced outward current. Despite this overestimation of the 8-pcpt-cGMP-induced current, the net outward current produced by 8 -pcpt-cGMP is much smaller in the presence of do- 
Control

$$
\text { 8-pcpt-cGMP }
$$

A

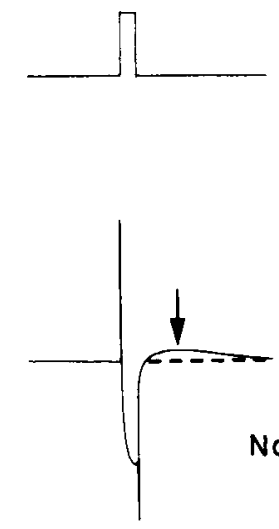

B

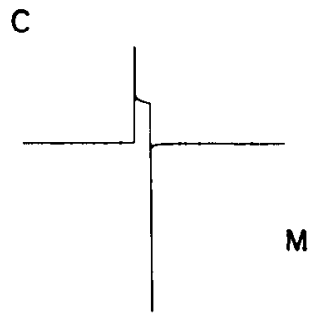

E

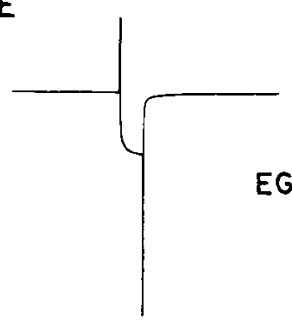

Mn

F

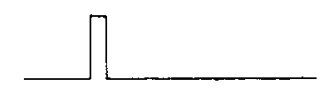

V

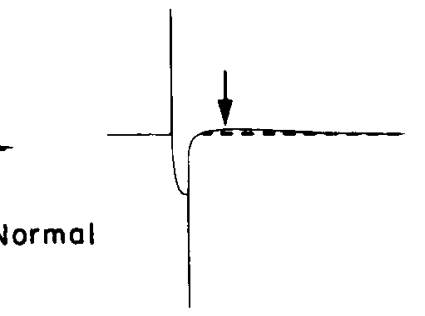

I

D
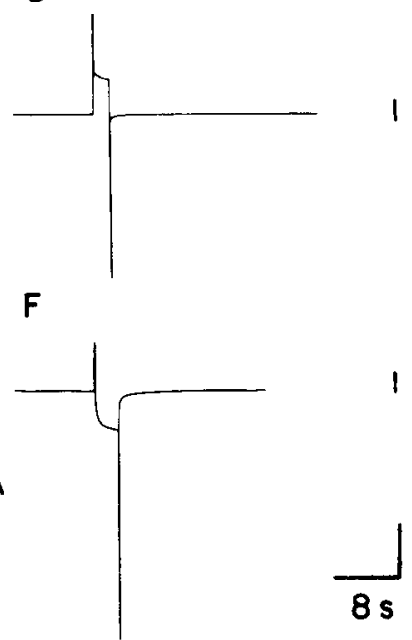

1

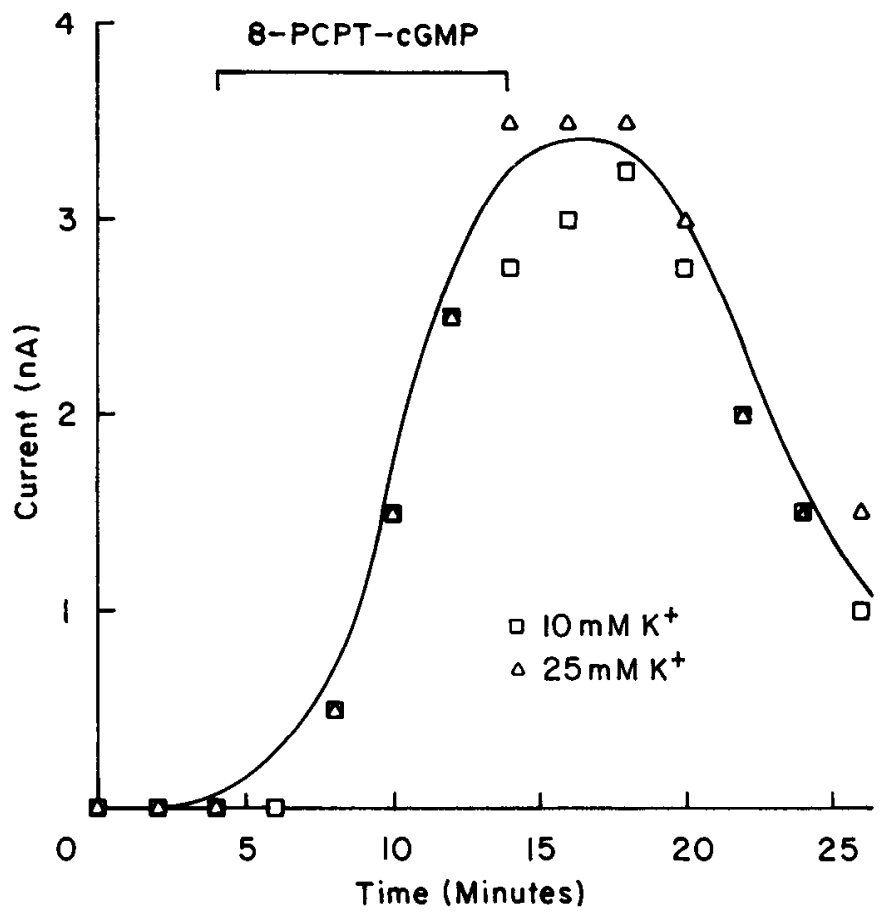

4
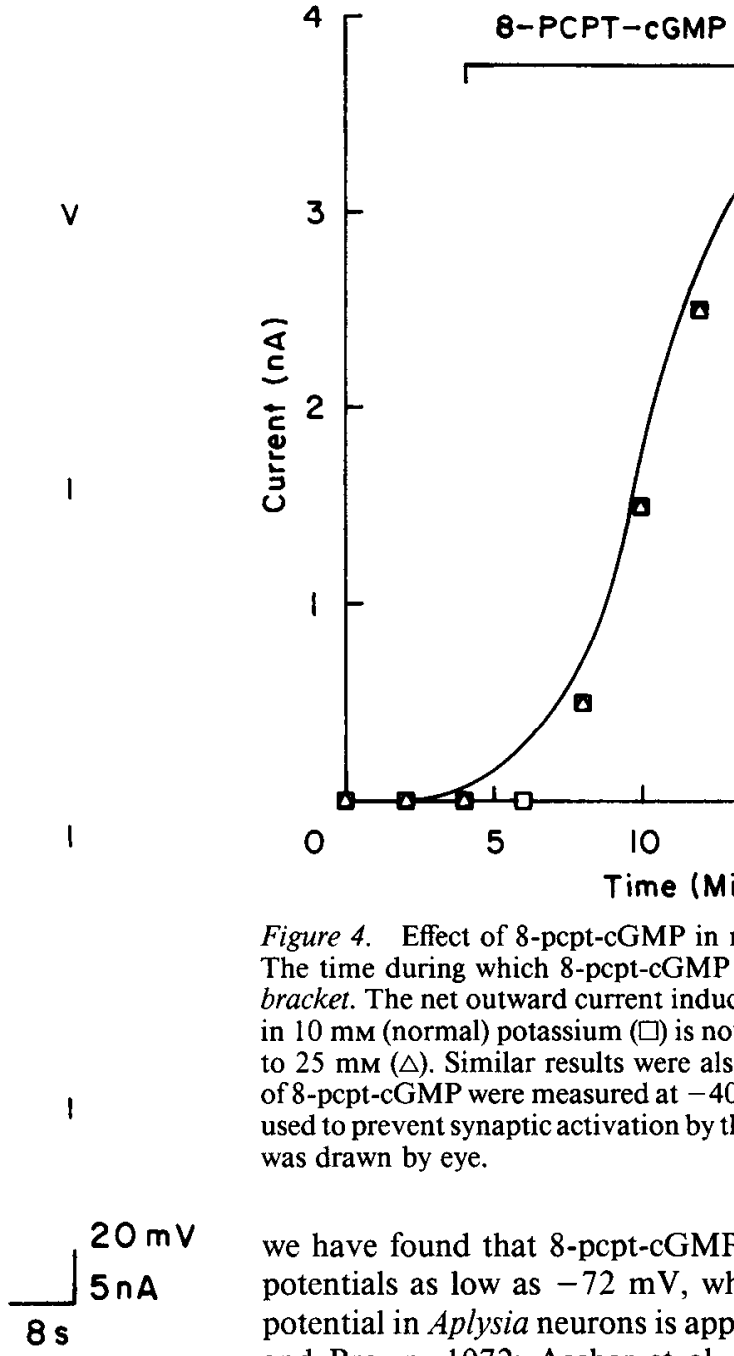

Figure 3. Effects of manganese and internal EGTA on the 8-pcptcGMP-induced reductions in the voltage-gated inward current and the outward tail current. $A$, Current (bottom) evoked by a $24 \mathrm{mV}$ depolarizing pulse (top) from $-70 \mathrm{mV}$. Arrow indicates slow tail current, which is outward compared with the holding current (dotted line). B, 8-pcptcGMP reduces the voltage-gated inward current and the slow outward tail current. $C$, After washout of the 8-pcpt-cGMP, manganese was substituted for calcium. Depolarization now evokes a net outward current. $D, 8$-pcpt-cGMP produces no change in the presence of manganese. $E$, Current evoked by a $22 \mathrm{mV}$ depolarization from $-70 \mathrm{mV}$ in an EGTA-loaded cell. $F$, 8-pcpt-cGMP reduces the voltage-gated inward current even in the presence of internal EGTA.

pamine than in its absence. Normally, 8-pcpt-cGMP gives similar responses with multiple applications (data not shown). Thus, dopamine appears to at least partially occlude the effects of 8-pcpt-cGMP. These data further support the hypothesis that 8-pcpt-cGMP decreases $I_{\mathrm{Ca}}$ in R15.

We cannot completely eliminate the possibility that 8-pcptcGMP increases chloride currents. This is because the long time course of application and removal of 8-pcpt-cGMP (i.e., $60 \mathrm{~min}$ ) allows chloride to redistribute during an experiment in which the chloride equilibrium potential is altered by lowering external chloride. However, it seems highly unlikely that 8-pcpt-cGMP acts on a chloride current. First, increasing chloride current should increase the slow outward tail current, whereas 8-pcptcGMP decreases the slow outward tail current. Furthermore,

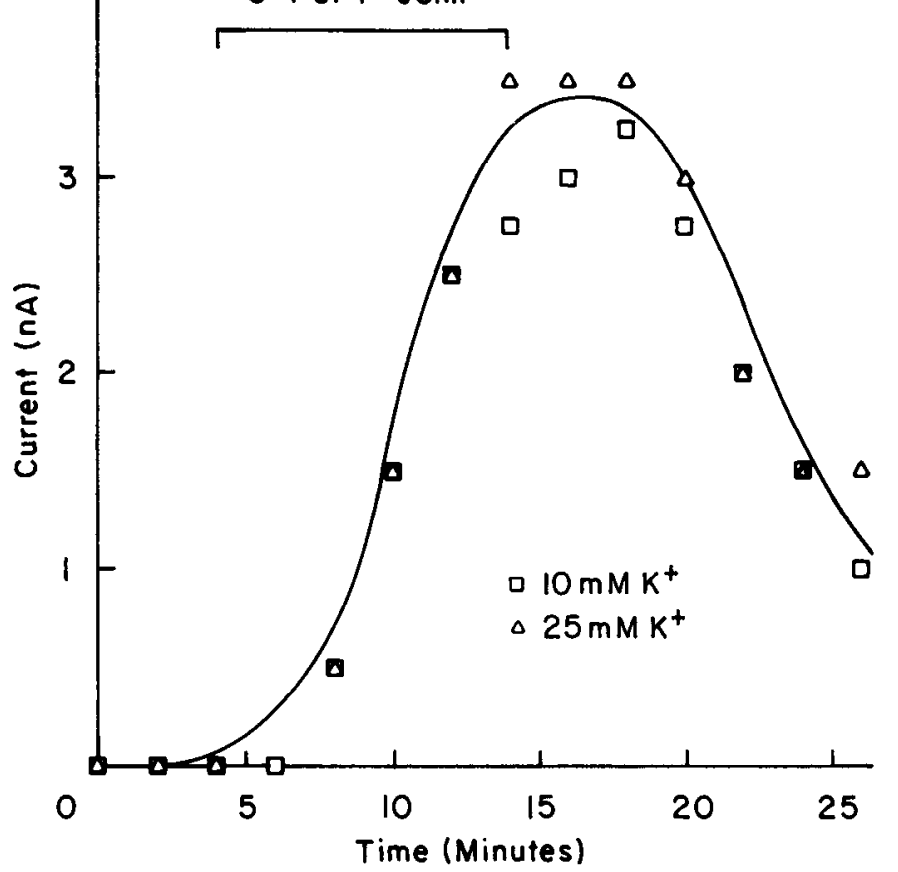

$\triangle 25 \mathrm{mM} \mathrm{K}^{+}$

Figure 4. Effect of 8-pcpt-cGMP in normal and elevated potassium. The time during which 8-pcpt-cGMP was applied is indicated by the bracket. The net outward current induced by 8 -pcpt-cGMP at $-29 \mathrm{mV}$ in $10 \mathrm{~mm}$ (normal) potassium $(\square)$ is not affected by elevating potassium to $25 \mathrm{~mm}(\triangle)$. Similar results were also obtained in cells where effects of 8-pcpt-cGMP were measured at $-40 \mathrm{mV}$. Low-calcium seawater was used to prevent synaptic activation by the elevated potassium. The curve was drawn by eye.

we have found that 8-pcpt-cGMP induces outward current at potentials as low as $-72 \mathrm{mV}$, while the chloride equilibrium potential in Aplysia neurons is approximately $-58 \mathrm{mV}$ (Russell and Brown, 1972; Ascher et al., 1976). Finally, no chloride channels have been reported to be blocked by manganese. Thus, we conclude that 8-pcpt-cGMP decreases $I_{\mathrm{Ca}}$.

\section{Analysis of 8-pcpt-cGMP effects in the context of bursting}

The finding that 8-pcpt-cGMP decreases $I_{\mathrm{Ca}}$ presents a quandry. Outward currents induced by transmitters or injected under current clamp lead to enhancement of the interburst hyperpolarization (Frazier et al., 1967; Wilson and Wachtel, 1978; Ayers and Selverston, 1979; Benson and Levitan, 1983). How can induction of tonic spiking be associated with a decrease in inward $I_{\mathrm{Ca}}$ ? A decrease in inward current is, after all, equivalent to increasing outward current. The voltage-clamp analysis presented thus far employs simple voltage paradigms where the effects of 8-pcpt-cGMP are analyzed under steady-state conditions. However, R15 usually is not at steady state. Rather, the cell produces oscillatory activity, and oscillatory systems are far from equilibrium and steady-state conditions. Thus, in order to gain an understanding of how 8-pcpt-cGMP altcrs bursting, a voltage-clamp paradigm was used in which trains of short spikelike depolarizations were alternated with $40 \mathrm{sec}$ periods at the holding potential. In effect, the cell was commanded to burst but at a spike frequency and interburst period set by the experimenter. While this paradigm does not exactly mimic endogenously generated bursting, it approximates it while still allowing for analysis of the effects of 8-pcpt-cGMP under voltage clamp. 


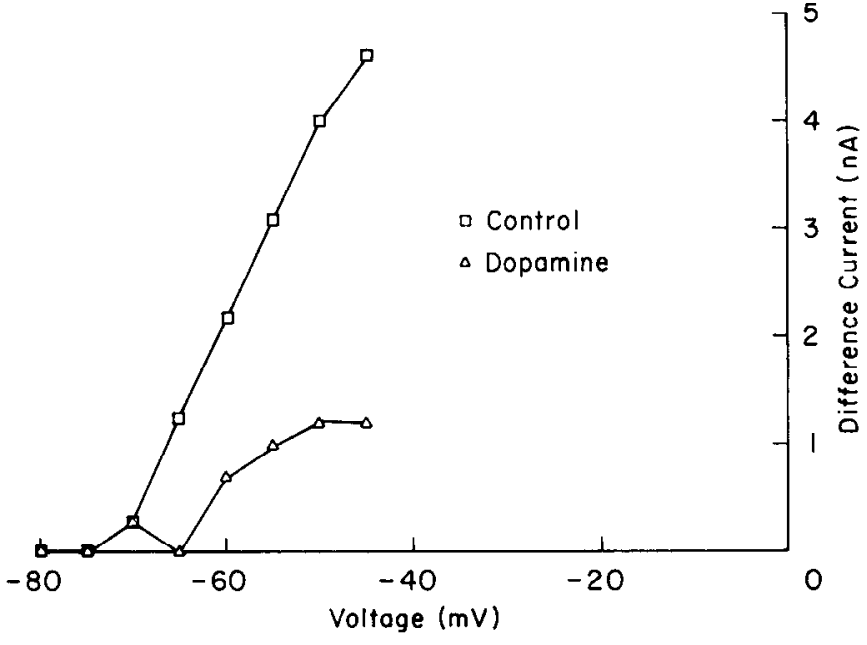

Figure 5. Dopamine reduces the 8-pcpt-cGMP-induced voltage-gated current. Shown are the 8-pcpt-cGMP-induced currents in the absence $(\square)$ and presence $(\triangle)$ of $0.5 \mathrm{~mm}$ dopamine. Difference curves were obtained by subtracting the $I-V$ curve in the presence of 8-pcpt-cGMP from the $I-V$ curve after washing out 8-pcpt-cGMP.

Action potentials and depolarizing pulses that cause calcium influx have been shown to produce 2 slow currents important for generating oscillatory activity in molluscan bursting cells. First, a calcium-activated inward current is seen that peaks approximately $500 \mathrm{msec}$ after an action potential (see upward arrows in Fig. 6). This current, which is thought to be due to the opening of nonselective cation channels in the axon of R15, contributes to maintaining the burst and underlies the depolarizing afterpotential seen after the last spike in the burst (Kramer and Zucker, 1985a; Adams and Levitan, 1985; Kramer, 1986). Second, at the end of a "burst" of depolarizing pulses, an outward current with slow kinetics is seen (see downward arrows in Fig. 6). This current, which underlies the interburst hyperpolarization, is due to calcium-dependent inactivation of subthreshold calcium current (Adams and Levitan, 1985; Kramer and Zucker, 1985b). It has been shown that, because of the large difference in the kinetics of these currents, the calciumactivated cation current after the first pulse in a train is relatively uncontaminated by the slow inactivation current. Likewise, the slow inactivation current seen 2-4 sec after the end of a burst is uncontaminated by the nonselective cation current, which decays more quickly (Adams, 1985).

The effects of 8-pcpt-cGMP on these voltage-clamp-induced "burst" currents in a representative cell are shown in Figure $6 B$. 8-pcpt-cGMP induces a number of changes. First, the holding current evident before each burst becomes more outward. This is consistent with the finding that 8-pcpt-cGMP decreases steadystate $I_{\mathrm{Ca}}$. Additionally, a $44 \%$ decrease in the pulse-evoked inward current is seen. This may simply be due to a decrease in $I_{\mathrm{Ca}}$, which, in turn, leads to a reduction in the calcium-activated nonselective cation current. On the other hand, 8-pcpt-cGMP might increase some opposing outward current or affect the cation channels directly. The predominant outward current active $500 \mathrm{msec}$ after an action potential is the calcium-activated potassium current. This current is blocked by low concentrations of tetraethylammonium (TEA; $K_{d}=0.4 \mathrm{~mm}$ ) (Hermann and Gorman, 1981). Figure $6, C$ and $D$, shows that 8-pcptcGMP reduces the spike-evoked inward current in the presence of concentrations of TEA that block greater than $92 \%$ of the calcium-activated potassium current, and thus 8-pcpt-cGMP does not act by increasing that current. The fact that the calciumactivated nonselective cation current is located predominantly in the axon of R15 prevented further study on its modulation by 8-pcpt-cGMP. Thus, we cannot determine whether 8-pcptcGMP decreases this current directly or indirectly, or whether some opposing current other than the calcium-activated potassium current is modulated. In either case, we are still left with the apparent paradox that conversion of bursting to beating activity by 8-pcpt-cGMP is accompanied by a decrease in inward currents. The reduction of $I_{\mathrm{Ca}}$ and spike-evoked calciumdependent inward current tends to remove the drive to spike vigorously and thus probably causes the decrease in spike frequency seen with 8-pcpt-cGMP.

In order to analyze fully the effects of 8-pcpt-cGMP one must also consider the effects of 8-pcpt-cGMP on the current terminating a burst. Normally, calcium-dependent inactivation of the subthreshold calcium current leads to the cessation of a burst (Adams and Levitan, 1985; Kramer and Zucker, 1985b). In fact, 8-pcpt-cGMP reduces by some $50 \%$ the slow outward tail current which reflects the inactivation of the calcium current. This is evident in experiments in which the slow outward tail current is evoked either by a long subthreshold depolarization or by trains of short spikelike depolarizations (see downward arrows in Figs. 3 and 6). This decrease in the current responsible for producing the interburst hyperpolarization leads to the shallow interbursts seen with 8-pcpt-cGMP.

Thus far, the currents underlying bursting have been analyzed in a context in which spike frequency is fixed. However, 8-pcptcGMP decreases the inward currents that control spike frequency. We therefore tested whether varying spike frequency alters the currents that produce bursting. Shown in Figure $7 \mathrm{~A}$ are the currents evoked by a train of pulses given with an interpulse interval of $0.5 \mathrm{sec}$ in a representative cell. Large inward, as well as outward, currents are seen. Doubling the interpulse interval does not affect the inward current seen after the first pulse (the only one nol contaminated by the slow outward current) and only reduces the peak inward current, seen after the third pulse, by $18 \%$. However, the slow outward tail current seen at the end of the train of pulses is reduced by $45 \%$ (Fig. $7 B$ ). A further decrease in the outward tail current is seen when the interpulse interval is increased to $1.5 \mathrm{sec}$, but once again the inward tail current after the first pulse is unchanged (Fig. $7 C$ ). These changes are evident in the plot shown in Figure $7 D$. There we see that increasing the interpulse interval, from a period commonly seen in control cells to a period seen in cells exposed to 8-pcpt-cGMP, markedly reduces the outward current responsible for terminating a burst. This finding is not surprising since increasing the interval between spikes decreases temporal summation of the outward current. Thus, combining the reduction in the slow outward current produced directly by 8 -pcptcGMP (Fig. 6), with the indirect reduction caused by decreasing spike frequency (Fig. 7), leads to a profound decrease in this outward current responsible for terminating the burst.

These results suggest that 8-pcpt-cGMP may act by the following mechanism to convert R15's activity from robust bursting to slow beating. 8-pcpt-cGMP decreases the subthreshold $I_{\mathrm{Ca}}$ and the action potential-induced inward and outward currents. The reduction of inward currents, in turn, causes a decrease in spike frequency that further diminishes the outward current responsible for terminating the burst. Reducing this out- 


\section{$+1+1 \quad 1 \quad 1 \quad 1$}

A
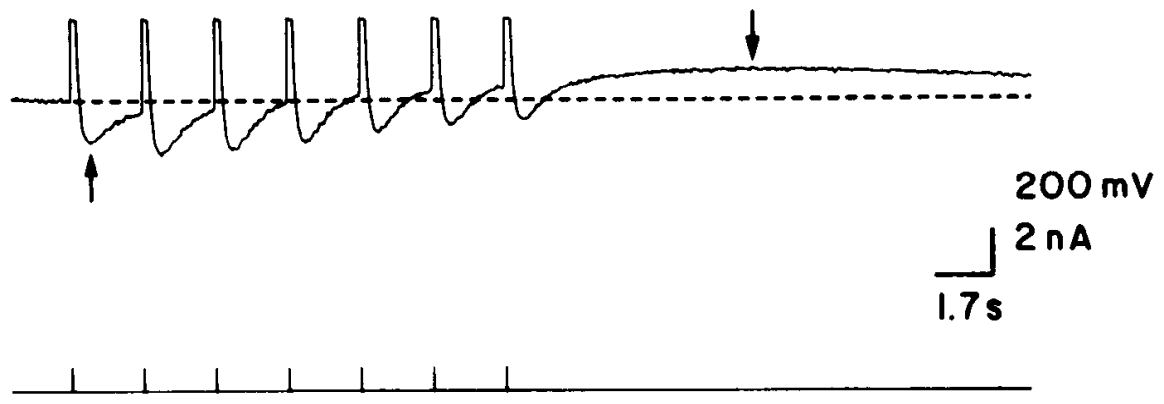

B
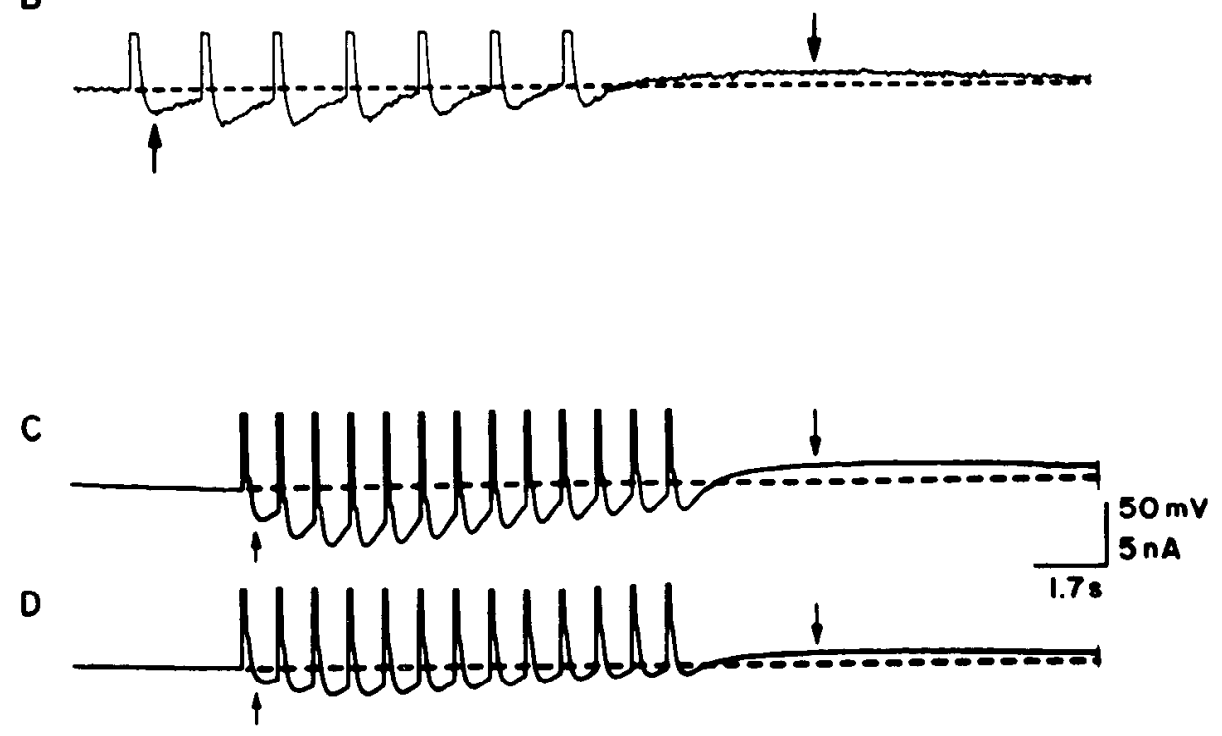

Figure 6. 8-pcpt-cGMP decreases the burst-evoked inward and outward currents. $A$, Trains of $10 \mathrm{msec}$ depolarizations (upper traces in $A$ and $B$ ) from $-50 \mathrm{mV}$ to $+50 \mathrm{mV}$ evoke inward tail currents (upward arrow), followed by a slow outward tail current (downward arrow) at the end of the simulated "burst" of depolarizations. B, Perfusion for $18 \mathrm{~min}$ with $0.85 \mathrm{~mm} 8$-pcptcGMP decreases the inward (upward arrow) and outward (downward arrow) tail currents. The holding current before the "burst" shifted $2.5 \mathrm{nA}$ outward in the presence of 8-pcpt-cGMP. $C$, Burst-evoked currents in another cell bathed in $5 \mathrm{~mm}$ TEA. $D$, A $10 \mathrm{~min}$ perfusion with $1.7 \mathrm{~mm}$ 8-pcpt-cGMP reduces both the inward and outward tail currents in the continued presence of TEA. The current before the "burst" shifted 4 nA outward. ward current decreases the interburst hyperpolarization. Eventually, the outward current becomes so small that it is insufficient to produce a long-lasting interburst hyperpolarization. The cell is left with steady-state voltage-gated and spike-evoked inward currents, which, although reduced, are still sufficient to maintain beating activity. If this scheme is correct, 8-pcpt-cGMP does not act simply to depolarize or hyperpolarize R15; rather, 8-pcptcGMP converts bursting to beating activity by reducing both the inward and outward currents required for bursting.

\section{8-pcpt-cGMP alters $R 15$ 's responses to inhibitory and excitatory input}

R 15 is bombarded by a wide variety of synaptic potentials when the nervous system is left intact (see Adams and Benson, 1985). In order to determine the physiological significance of 8-pcptcGMP-induced beating activity, one must determine whether 8-pcpt-cGMP changes R15's responsiveness to synaptic input. As a first step in this direction, the effects of injected current pulses were compared in the absence and presence of 8-pcptcGMP. While these stimuli are not physiological, applying current directly to $\mathrm{R} 15$ avoids the need to correct for presynaptic effects. Presumably, some synaptic currents will produce effects similar to those seen with current pulses.

Shown in Figure 8 are the responses of R 15 to hyperpolarizing and depolarizing current pulses. Very little change in the basal bursting behavior is seen with either small depolarizing or hy- perpolarizing current pulses, delivered either during the burst or during the interburst hyperpolarization (Fig. 8, $A, B$ ). 8-pcptcGMP was then applied to the cell, and the effects of identical pulses were determined. A hyperpolarizing current pulse now initiates a long-lasting hyperpolarization (Fig. $8 \mathrm{C}$ ), presumably because the hyperpolarizing pulse turns off the small amount of voltage-gated $I_{\mathrm{Ca}}$ available to depolarize the cell. Dramatic hyperpolarization is also seen with depolarizing pulses which increase spike frequency, allow calcium entry, and thus increase the calcium-dependent inactivation of the small amount of resting $I_{\mathrm{Ca}}$ (Fig. 8, $D, E$ ). Current pulses also can sometimes induce dampened bursting activity (Fig. $8 F$ ). These results demonstrate that the 8-pcpt-cGMP-induced reduction of subthreshold currents that underlie bursting activity makes $R 15$ very sensitive to perturbation. A physiological stimulus that produces 8-pcptcGMP-like effects would therefore not only alter basal bursting activity, but would also effectively sensitize R15 to synaptic input.

\section{Discussion}

We have found that 8-pcpt-cGMP can convert cell R15's bursting activity into slow beating activity. Voltage-clamp studies reveal that 8-pcpt-cGMP reduces the steady-state subthreshold $I_{\mathrm{Ca}}$. Furthermore, voltage-clamp paradigms that mimic bursting activity demonstrate that the spike-evoked inward and outward currents that underlie bursting are also decreased. The decreases 


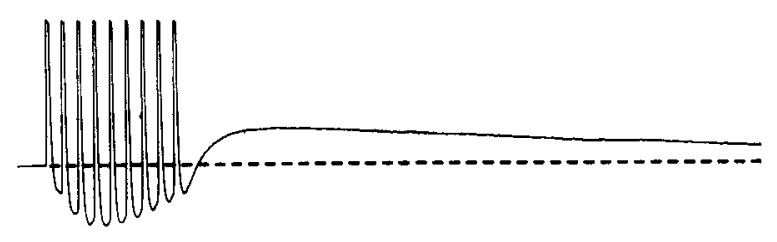

8

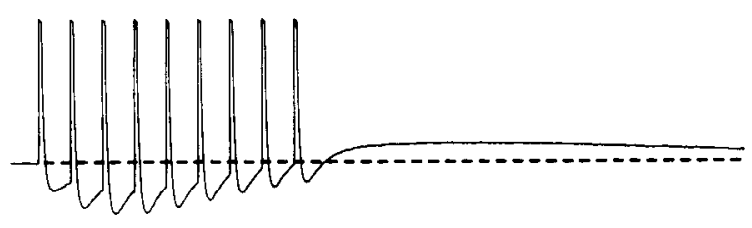

C

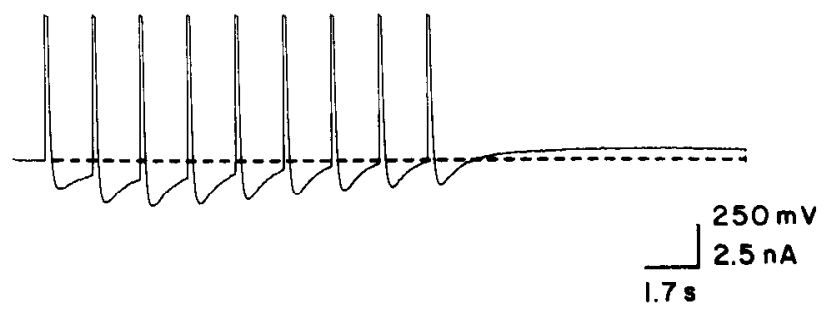

D

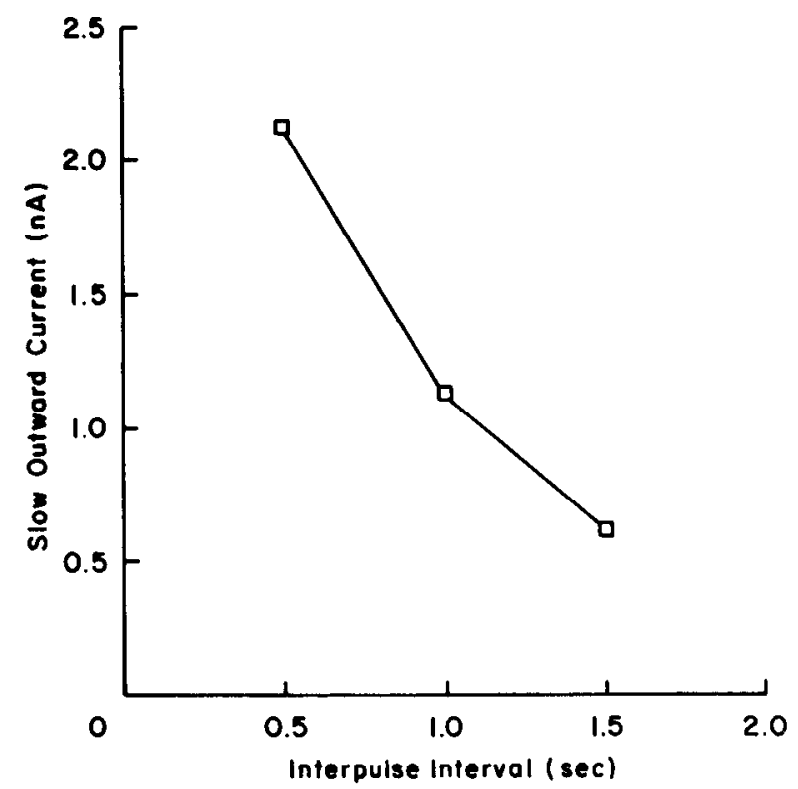

Figure 7. Effect of varying the interpulse interval on the slow outward tail current following a simulated burst produced as described for Figure 6. Top trace in each pair is the train of voltage pulses; bottom trace, evoked current. $A$, Currents evoked by a train of pulses with an interpulse interval of $0.5 \mathrm{sec} . B$, Currents evoked with interpulse interval of $1.0 \mathrm{sec}$. $C$, Currents evoked with interpulse interval of 1.5 sec. $D$, Effect of interpulse interval on the peak slow outward tail current. Note that increasing the interpulse interval dramatically reduces the slow outward tail current.

in these 2 currents may be due merely to the fact that they are calcium dependent and thus respond indirectly to the reduction of $I_{\mathrm{Ca}}$. These findings suggest that 8-pcpt-cGMP does not simply hyperpolarize or depolarize R15 but rather dampens bursting. The cell beats slowly because inward currents are too small to drive high-frequency spiking, and the outward current is too small to produce an interburst hyperpolarization.

To test this hypothesis we employed a computer model originally formulated to demonstrate that the spike-evoked currents, when combined with a steady-state inward current, are sufficient to produce bursting activity (Adams, 1985). When physiological parameters are given for the resting steady-state current and the spike-evoked tail currents, a bursting pattern reminiscent of that in R15 is produced (results not shown). Reducing both the inward and outward currents by $25 \%$ leads to production of bursts with lower spike frequencies and shallow interbursts similar to those seen in Figure $1 C$. Further reduction of the burst currents to $50 \%$ of their initial values gives tonic low-frequency beating activity. Thus, decreasing the opposing inward and outward burst currents to a degree seen with 8-pcpt-cGMP leads the model to produce the same activity seen with the cyclic GMP analog. This suggests that the hypothesis that bursting activity can be converted to beating activity by reducing both the inward and outward currents required for production of oscillatory activity is a viable one.

Further support for this hypothesis is provided by examining the response of R15 to stimulation of the branchial nerve. This elicits an inhibitory synapse (Parnas et al., 1974) that appears to reduce the same currents in R15 as does 8-pcpt-cGMP, albeit to a larger extent (Adams et al., 1980; Adams and Benson, 1985; R. Kramer, personal communication). Recovery from the synaptic hyperpolarization is associated with dampened bursting, and this pattern of activity displays many of the features seen in the presence of 8-pcpt-cGMP (Parnas et al., 1974; E. S. Levitan and I. B. Levitan, unpublished observations). This result suggests that conversion of a burster to a slow beater can occur with physiological stimuli that reduce opposing burst currents.

The dampening of bursting produced by 8-pcpt-cGMP also amplifies R15's responsiveness to hyperpolarizing currents that are either injected experimentally or evoked by increasing spike frequency. 'I his change most likely arises because the cyclic GMP analog decreases the inward currents that normally oppose a hypcrpolarization and bring the cell back to the spike threshold. Synaptic potentials may likewise produce larger alterations 
A
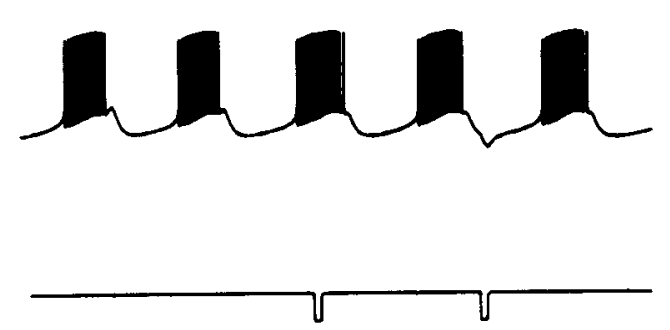

C
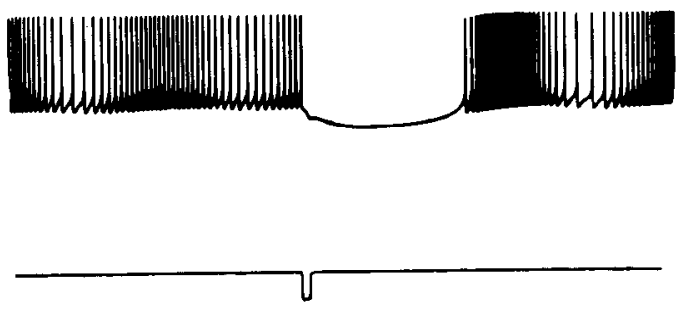

$E$

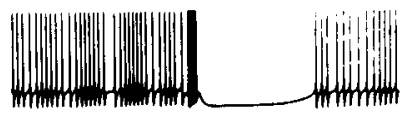

B

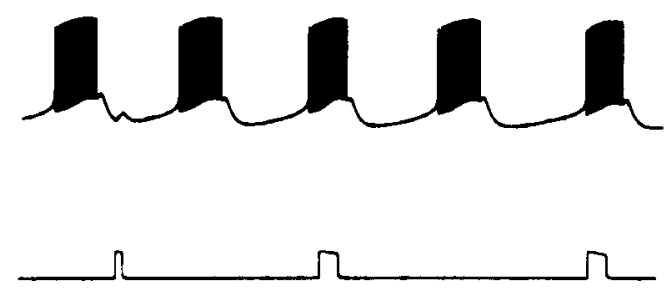

D

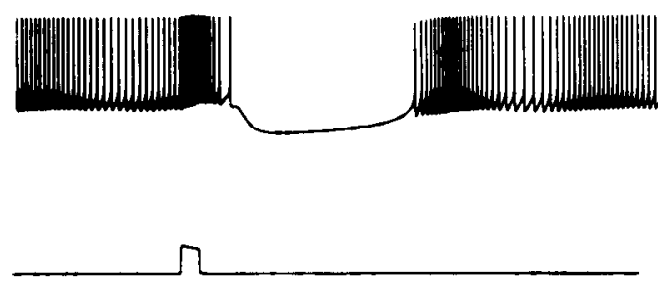

$\mathbf{F}$

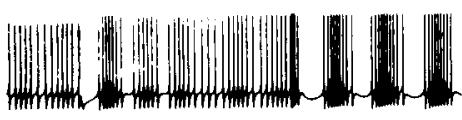

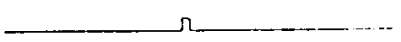

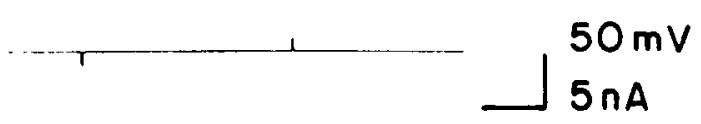

Figure 8. 8-pcpt-cGMP alters the responsiveness of R15 to inhibitory and excitatory currents. A, Hyperpolarizing current pulses (lower trace), delivered during either the burst or interburst, produce little effect on R15's bursting activity (upper trace). $B$, Similarly, depolarizing current pulses do not alter the basal bursting activity. After induction of beating activity by 8-pcpt-cGMP, a hypcrpolarizing current pulse $(C)$ or a depolarizing pulse $(D)$ can both induce a long-lasting hyperpolarization. $E$, Same as $D$ in another cell (tops of spikes clipped off). $F$, Same cell as $E$. Shorter hyperpolarizing or depolarizing pulses can trigger transient periods of bursting. Eventually, resumption of beating activity is seen. Time calibration: $8 \mathrm{sec}, A-D ; 20 \mathrm{sec}, E$ and $F$.

in R15's activity in the presence of 8-pcpt-cGMP-like stimuli. This is reminiscent of the effects of 5-HT, which acts via cyclic AMP to alter R15's responsiveness to current pulses (Levitan and Levitan, 1988). While 8-pcpt-cGMP and cyclic AMP act differently, both modulate opposing currents and produce effects that cannot be termed simply excitatory or inhibitory. Both serve also to illustrate that modulatory mechanisms, which produce straightforward changes in activity in normally silent cells that are near equilibrium as far as the membrane potential is concerned, can produce complicated behavior in an oscillatory system such as a neuronal burster.

It is interesting that cyclic AMP and 8-pcpt-cGMP produce apparently opposite effects on the subthreshold $I_{\mathrm{Ca}}$. We cannot be certain that the effects of 8-pcpt-cGMP represent the physiological action of cyclic GMP. However, 8-position-substituted cyclic GMP analogs have been shown to mimic some actions of cyclic GMP (Miller et al., 1973; Zimmerman et al., 1985). Thus, it seems possible that cyclic AMP increases and cyclic GMP decreases $I_{\mathrm{Ca}}$ in R15. A similar situation occurs in heart, where cyclic GMP activates a phosphodiesterase that, in turn, lowers cyclic AMP and thus decreases $I_{\mathrm{Ca}}$ (Hartzell and Fischmeister, 1986). However, it is unlikely that 8-pcpt-cGMP acts by stimulating a phosphodiesterase in R15. First, 8-positionsubstituted cyclic GMP analogs are poor activators of the cyclic GMP-dependent phosphodiesterase (Erneux et al., 1985). More important, no change in $I_{\mathrm{R}}$ is evoked by 8 -pcpt-cGMP. This current, like $I_{\mathrm{Ca}}$, is modulated by cyclic AMP (Drummond et al., 1980; Benson and Levitan, 1983; Lemos and Levitan, 1984), and thus a change in cyclic AMP concentration would be expected to affect it also. Of course, this argument assumes that cyclic GMP-activated phosphodiesterase, calcium channels, and inwardly rectifying potassium channels are evenly distributed throughout the cell.

8-pcpt-cGMP might also act by activating cyclic GMP-dependent protein kinase, which occurs in the Aplysia nervous system (Novak-Hofer et al., 1985). On the other hand, it might modulate channel gating in an ATP-independent manner, as it does in vertebrate rods (Haynes et al., 1986; Zimmerman and Baylor, 1986). It is unlikely that 8-pcpt-cGMP alters bursting by cross-reacting with cyclic AMP-dependent protein kinase or by inhibiting a phosphodiesterase since the effects of 8-pcptcGMP differ from those of cyclic AMP. In any case, the recent finding that cyclic GMP acts via cyclic GMP-dependent protein kinase to increase $I_{\mathrm{Ca}_{\mathrm{a}}}$ in certain Helix neurons (Paupardin-Tritsch 
et al., 1986a, b) suggests that cyclic GMP, like cyclic AMP, produces varying effects in different cell types. This is reinforced by the fact that cyclic GMP increases a sodium current in some molluscan neurons (Connor and Hockberger, 1984).

The effects of 5-HT and 8-pcpt-cGMP on R 15 suggest that a burster can be converted into a beater by at least 2 mechanisms. 5-HT, $50 \mu \mathrm{M}$, increases the subthreshold $I_{\mathrm{Ca}}$ so that the cell always returns to the action potential threshold (Levitan and Levitan, 1988), whereas 8-pcpt-cGMP dampens oscillatory activity until the cell is left beating slowly. These changes in activity may also alter the cell's responsiveness to synaptic input. While we have not determined whether cyclic GMP is a physiological second messenger in R15, it is interesting that the conversion of cell 11 in Otala from beating to bursting activity by vasopressin is associated with an increase in steady-state inward current. Injection of inward current, however, does not produce bursting (Barker and Smith, 1976). Perhaps, in analogy to recovery of bursting seen when washing away 8-pcpt-cGMP from R 15, bursting is induced in cell 11 by increasing the steadystate inward current and the action potential-evoked outward current. This mechanism may also be important for interconversion between bursting and beating activity in cells that are not well suited for voltage-clamp analysis (Grace and Bunney, 1984; Marder and Eisen, 1984; Dekin et al., 1985). For example, in nigral dopamine neurons, exposure to the excitatory transmitter glutamate shifts the cells from a beating to a bursting state (Grace and Bunney, 1984). It will be of interest to extend the present analysis to some of these other systems to determine the generality of these ionic mechanisms.

\section{References}

$\Lambda$ dams, W. B. (1985) Slow depolarizing and hyperpolarizing currents which mediate bursting in Aplysia neurone R15. J. Physiol. (Lond.) 360: 51-68.

Adams, W. B., and J. A. Benson (1985) The generation and modulation of endogenous rhythmicity in the Aplysia bursting pacemaker neurone R 15. Prog. Biophys. Mol. Biol. 46: 1-49.

Adams, W. B., and I. B. Levitan (1985) Voltage and ion dependences of the slow currents which mediate bursting in Aplysia neurone R15. J. Physiol. (Lond.) 360: 69-93.

Adams, W. B., I. Parnas, and I. B. Levitan (1980) Mechanism of longlasting synaptic inhibition in Aplysia neuron R15. J. Neurophysiol. 44: $1148-1160$.

Alving, B. O. (1968) Spontaneous activity in isolated somata of Aplysia pacemaker neurons. J. Gen. Physiol. 51: 29-45.

Ascher, P., D. Kunze, and T. O. Neild (1976) Chloride distribution in Aplysia neurones. J. Physiol. (Lond.) 256: 441-464.

Ayers, J. L., Jr., and A. I. Selverston (1979) Monosynaptic entrainment of an endogenous pacemaker network: A cellular mechanism for von Holst's magnet effect. J. Comp. Physiol. 129: 5-17.

Barker, J. L., and T. G. Smith (1976) Peptide regulation of neuronal membrane properties. Brain Res. 103: 167-170.

Benson, J. A., and I. B. Levitan (1983) Serotonin increases an anomalously rectifying $\mathrm{K}^{+}$current in the Aplysia neuron R15. Proc. Natl. Acad. Sci. USA 80: 3522-3525.

Carpenter, D. O. (1982) Cellular Pacemakers, Vol. 1, Wiley, New York.

Connor, J. A., and P. Hockberger (1984) A novel membrane sodium current induced by injection of cyclic nucleotides into gastropod neurones. J. Physiol. (Lond.) 354: 139-162.

Dekin, M. S., G. B. Richerson, and P. A. Getting (1985) Thyrotropinreleasing hormone induces rhythmic bursting in neurons of the nucleus tractus solitarius. Science 229:64-69.

Drummond, A. H., J. A. Benson, and I. B. Levitan (1980) Serotonininduced hyperpolarization of an identified Aplysia neuron is mediated by cyclic AMP. Proc. Natl. Acad. Sci. USA 77: 5013-5017.

Dutton, A., and R. E. J. Dyball (1979) Phasic firing enhances vaso- pressin release from the rat neurohypophysis. J. Physiol. (Lond.) 290: 433-440.

Erneux, C., F. Miot, P. J. M. Van Haastert, and B. Jastorff (1985) The binding of cyclic nucleotide analogs to a purified cyclic GMP-stimulated phosphodiesterase from bovine adrenal tissue. J. Cyclic Nucleotide Protein Phos. Res. 10: 463-472.

Frazier, W. T., E. R. Kandel, I. Kupfermann, R. Waziri, and R. E. Coggeshall (1967) Morphological and functional properties of identified neurons in the abdominal ganglion of Aplysia californica. $\mathrm{J}$. Neurophysiol. 30: 1288-1351.

Gillary, M. L., and D. Kennedy (1969) Neuromuscular effects of impulse pattern in a crustacean motoneuron. J. Neurophysiol. 32: 595606.

Gorman, A. L. F., A. Hermann, and M. V. Thomas (1982) Ionic requirements for membrane oscillations and their dependence on the calcium concentration in a molluscan pace-maker neurone. J. Physiol. (Lond.) 327: 185-217.

Grace, A. A., and B. S. Bunney (1984) The control of firing pattern in nigral dopamine neurons: Burst firing. J. Neurosci. 4: 2877-2890.

Harris-Warrick, R. M., and R. E. Flamm (1986) Chemical modulation of a small central pattern generator circuit. TINS 9: 432-436.

Hartzell, H. C., and R. Fischmeister (1986) Opposite effects of cyclic GMP and cyclic AMP on $\mathrm{Ca}^{2+}$ current in single heart cells. Nature 323: 273-275.

Haynes, L. W., A. R. Kay, and K. W. Yau (1986) Single cyclic GMPactivated channel activity in excised patches of rod outer segment membrane. Nature 321: 66-70.

Hermann, A., and A. L. F. Gorman (1981) Effects of tetraethylammonium on potassium currents in a molluscan neuron. J. Gen. Physiol. 78: $87-110$.

Kramer, R. H. (1986) Axonal contribution to subthreshold currents in Aplysia bursting pacemaker neurons. Cell. Mol. Neurobiol. 6: 239253.

Kramer, R. H., and R. S. Zucker (1985a) Calcium-dependent inward current in Aplysia bursting pace-maker neurones. J. Physiol. (Lond.) 362: $107-130$.

Kramer, R. H., and R. S. Zucker (1985b) Calcium-induced inactivation of calcium current causes the interburst hyperpolarization of Aplysia bursting pacemaker neurones. J. Physiol. (Lond.) 362:131160.

Lemos, J. R., and I. B. Levitan (1984) Intracellular injection of guanyl nucleotides alters the serotonin-induced increase in potassium conductance in Aplysia neuron R15. J. Gen. Physiol. 83: 269-285.

Levitan, E. S., and I. B. Levitan (1988) Serotonin acting via cyclic AMP enhances both the hyperpolarizing and depolarizing phases of bursting pacemaker activity in the Aplysia neuron R15. J. Neurosci. 8: $1152-1161$.

Levitan, I. B., and J. Norman (1980) Different effects of cAMP and cGMP derivatives on the activity of an identified neuron: Biochemical and electrophysiological analysis. Brain Res. 187: 415-429.

Lewis, D. V., G. B. Evans, and W. A. Wilson (1984) Dopamine reduces slow outward current and calcium influx in burst firing neuron R15 of Aplysia. J. Neurosci. 4: 3014-3020.

Marder, E., and J. S. Eisen (1984) Electrically coupled pacemaker neurons respond differently to same physiological inputs and neurotransmitters. J. Neurophysiol. 51: 1362-1374.

Meyer, R. B., and J. P. Miller (1974) Aualogs of cyclic AMP and cyclic GMP: General methods of synthesis and the relationship of structure to enzymic activity. Life Sci. 14: 1019-1040.

Miller, J. P., K. H. Boswell, K. Muneyama, L. N. Simon, R. K. Robins, and D. A. Shuman (1973) Synthesis and biochemical studies on various 8-substituted derivatives of guanosine $3^{\prime}, 5^{\prime}$-cyclic phosphate, inosine $3^{\prime} 5^{\prime}$-cyclic phosphate xanthosine $3^{\prime}, 5^{\prime}$-cyclic phosphate. Biochemistry $12: 5310-5319$.

Novak-Hofer, I., J. R. Lemos, M. Villermain, and I. B. Levitan (1985) Calcium and cyclic nucleotide dependent protein kinases and their substrates in the Aplysia nervous system. J. Neurosci. 5: 151-159.

Parnas, I., D. Armstrong, and F. Strumwasser (1974) Prolonged excitatory and inhibitory synaptic modulation of a bursting pacemaker neuron. J. Neurophysiol. 37: 594-608.

Paupardin-Tritsch, D., P. Deterrc, and M. M. Gerschenfeld (1981) Relationship between two voltage-dependent serotonin responses of molluscan neurones. Brain Res. 217: 201-206.

Paupardin-Tritsch, D., C. Hammond, and H. M. Gerschenfeld (1986a) Serotonin and cyclic GMP both induce an increase of the calcium 
current in the same identified molluscan neurons. J. Neurosci. 6: $2715-2723$.

Paupardin-Tritsch, D., C. Hammond, H. M. Gerschenfeld, A. C. Nairn, and P. Greengard (1986b) cGMP-dependent protein kinase enhances $\mathrm{Ca}^{2+}$ current and potentiates the serotonin-induced $\mathrm{Ca}^{2+}$ current increase in snail neurons. Nature 323: 812-814.

Rapp, P. E. (1979) An atlas of cellular oscillators. J. Exp. Biol. 81: 281-306.

Roth, J. D., K. Lukowiak, and R. W. Berry (1984) Long-lasting inhibition of neuron R 15 of Aplysia: Role of the interneuron II network. Comp. Biochem. Physiol. 78A: 83-89.

Ruben, P., J. W. Johnson, and S. Thompson (1986) Analysis of FMRFamide effects on Aplysia bursting neurons. J. Neurosci. 6: 252-259.

Russell, J. M., and A. M. Brown (1972) Active transport of chloride by the giant neuron of the Aplysia abdominal ganglion. J. Gen. Physiol. 60: 499-518.

Selverston, A. I., and M. Moulins (1985) Oscillatory neural networks. Annu. Rev. Physiol. 47: 29-48.

Wilson, W. A., and H. Wachtel (1978) Prolonged inhibition in burst firing neurons: Synaptic inactivation of the slow regenerative inward current. Science 202: 772-775.

Zimmerman, A. L., and D. A. Baylor (1986) Cyclic GMP sensitive conductance of retinal rods consists of aqueous pores. Nature 321: 70-72.

Zimmerman, A. L., G. Yamanaka, F. Eckstein, D. A. Baylor, and I. Stryer (1985) Interaction of hydrolysis resistant analogs of cyclic GMP with the phosphodiesterase and light sensitive channel of retinal rod outer segments. Proc. Natl. Acad. Sci. USA 82: 8813-8817. 